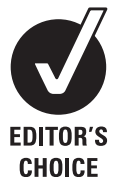

CHOICE

\title{
Understanding the link between environmental exposures and health: does the exposome promise too much?
}

\author{
Annette Peters, ${ }^{1,2}$ Gerard Hoek, ${ }^{3}$ Klea Katsouyanni ${ }^{4}$
}

${ }^{1}$ Helmholtz Zentrum

München-German Research Center for Environmental Health, Institute of Epidemiology II, Neuherberg, Germany ${ }^{2}$ Harvard School of Public Health, Department of Environmental Health, Boston, Massachusetts, USA

${ }^{3}$ Institute of Risk Assessment Sciences, University Utrecht, Utrecht, The Netherlands ${ }^{4}$ Department of Hygiene and Epidemiology, University of Athens Medical School, Athens, Greece

\section{Correspondence to \\ Professor Annette Peters, Institute of Epidemiology II, Helmholtz Zentrum München, Ingolstädter Landstr 1 . Neuherberg 85764, Germany: peters@helmholtz-muenchen. de}

Accepted 12 October 2011

Published Online First

11 November 2011

\begin{abstract}
Environmental exposures affecting human health range from complex mixtures, such as environmental tobacco smoke, ambient particulate matter air pollution and chlorination by products in drinking water, to hazardous chemicals, such as lead, and polycyclic aromatic hydrocarbons, such as benz(a)pyrene. The exposome has been proposed to complement the genome and be the totality of all environmental exposures of an individual over his or her lifetime. However, if measurements of the exposome in biological samples are the sole tool for exposure assessment there are a number of limitations. First, it has limited utility for fully capturing the impact of complex mixtures such environmental tobacco smoke or particulate matter air pollution. Second, a number of relevant environmental exposures such as noise, heat or electromagnetic fields do not have direct correlates as metabolites or protein adducts, but there is important evidence linking them with health effects. Third, functional genomic changes are likely in many instances to be both a susceptibility factor and a marker of internal doses in response to environmental exposures. Fourth, internal dose measurements of environmental exposures might have lost the distinct signature of the relevant sources. This paper emphasises the obligation of environmental epidemiology to provide robust evidence to assist timely and sufficient protection of vulnerable subgroups of populations from environmental hazards. Therefore, in applying the exposome concept to environmental health problems, a strong link with the external environment needs to be maintained.
\end{abstract}

Environmental exposures affecting human health range from complex mixtures, such as environmental tobacco smoke, ambient particulate matter air pollution and chlorination by products in drinking water, to hazardous chemicals, such as lead, and polycyclic aromatic hydrocarbons, such as benz(a)pyrene. Environmental epidemiology has been influential over the past decades characterising the link between exposures and health effects jointly with toxicological assessments. Epidemiology is particularly powerful when complex mixtures, for example, environmental tobacco smoke $^{1}$ and ambient particulate air pollution ${ }^{2}$ are concerned. Abatement strategies have been implemented with substantial gain for public health, although the exact mechanisms of action and the role of the specific chemophysical properties responsible for the health effects are still under investigation. ${ }^{3}$ In addition, environmental epidemiology might be a powerful tool when the critical mechanisms at play in vulnerable populations are not well accessible by animal or in-vitro experiments. $^{2}$

\section{THE DIFFICULTY OF MONITORING INTERNAL DOSES OF ENVIRONMENTAL EXPOSURES}

Environmental exposures exhibit a number of direct and indirect effects that induce health responses. Immediate health effects occur either as a consequence of high doses of exposure or in vulnerable individuals. Environmental exposures in addition contribute to more general pathophysiological mechanisms, and increase the burden and potential health effects through repeated and prolonged exposures by influencing the development of disease over the life course of individuals. For example, the indirect effects of ambient particulate matter such as increased inflammation, increased prothrombotic states and altered autonomic function have been identified as reasons for the cardiovascular disease burden of fine particles. ${ }^{2}$ Biomarkers describing changes of physiological states upon exposures to environmental agents are therefore frequently both indicators of internal effective doses of mixtures and early physiological responses at the same time (figure 1). Many of the environmental exposures of primary concern for public health actually lack specific biomarkers of internal dose that fully reflect the exposure. This still applies to environmental exposures that are complex mixtures, such as environmental tobacco smoke, particulate air pollution and photochemical smog. Furthermore, some environmental exposures have no direct correlate as a biomarker of internal dose, such as noise, heat stress and electromagnetic fields. In contrast, exposures to heavy metals, air toxins and endocrine disruptors may be monitored with extremely sophisticated analytical equipment in bodily fluids, in metabolic states, or as adducts. ${ }^{4}$

\section{EARLY PHYSIOLOGICAL RESPONSES TO ENVIRONIMENTAL EXPOSURES}

Important pathways that are affected by multiple environmental exposures and can be well characterised by biomarker panels include markers of oxidative stress, inflammation, altered immune responses and hormonal regulation ${ }^{2} 5$ among others. Some of the links highlighted in figure 1 have been well documented, whereas others are still under investigation.

Current advances in studying the molecular bases for disease development and progression are utilising high throughput techniques and 


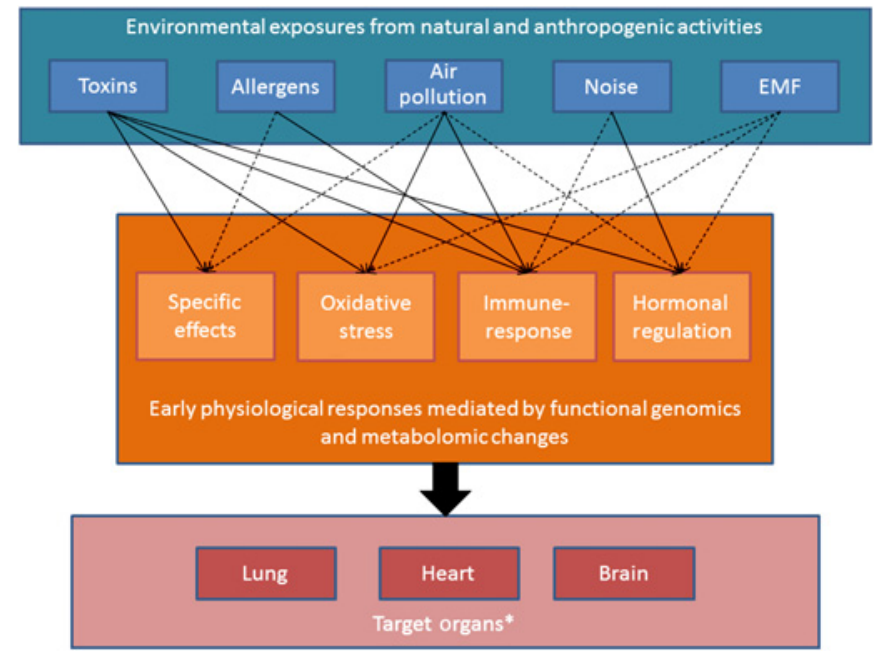

Figure 1 Schematic overview on a paradigm of environmental epidemiology linking traditional research and emerging biomarkers and altered functional genomics. Documented associations are shown as solid lines while hypothetical associations are shown as dashed lines. *Named target organs are those for which the major impact of the environment is expected, but multiple other organs such as liver, bladder, kidney and reproductive systems among others may be affected. Electro Magnetic Fields (EMF).

hypotheses-free approaches for data analyses. This research has revolutionised the understanding of the link between single nucleotide polymorphisms and common chronic diseases as well as their related quantitative traits based on whole genomeassociation studies. Efforts enlarging these concepts to functional genomics, including whole genome epigenomics and transcriptomics, are underway. ${ }^{6}$ Mapping the consequences of genetic regulation by characterising the metabolome has started to provide important novel insights into the role of genetic loci in determining of metabolite profiles. ${ }^{8}$ In consequence, these techniques call for integration into environmental epidemiology. ${ }^{9}$

\section{THE EXPOSOME-AN ADEOUATE INSTRUMENT FOR EXPOSURE ASSESSMENT?}

The exposome has been proposed to complement the genome and be the totality of all environmental exposures of an individual over his or her lifetime. ${ }^{4} 1011$ Recent advances in biological chemistry allow the characterisation of metabolites and protein adducts originating from environmental exposures, and thereby provide novel insights into internal effective doses of environmental exposures, which have long been precluded from studying. Rappaport and Smith ${ }^{10}$ have proposed, in a visionary fashion, that integrating these novel techniques into environmental epidemiology will advance science substantially. Recent discoveries using lipidomics for characterising links between genomic loci and lipid ratios ${ }^{8}$ highlight the potential utility for targeted metabolomics and call for the inclusion xenobiotics and their metabolites in these approaches. Also, the study of protein adducts is likely to unravel new insights into the modes of action of a number of environmental agents.

However, if this approach is used as the sole tool for exposure assessment we see a number of limitations. First, it has limited utility for fully capturing the impact of complex mixtures such as environmental tobacco smoke or particulate matter air pollution by reducing their characterisation to a set of measurable metabolites and adducts. In recent European burden of disease assessments, these complex mixtures in particular were associated with the largest loss in disability-adjusted life-years. ${ }^{12}$ Second, a number of relevant environmental exposures such as noise, heat or electromagnetic fields do not have direct correlates as metabolites or protein adducts, but there is important evidence linking them with health effects. Third, functional genomic changes are likely in many instances to be both a susceptibility factor and a marker of internal doses in response to environmental exposures. For example, oxidative stress is changing epigenetic markers; however, unrelated early life exposures or transgenerational modifications may result in similar epigenomic variation. Therefore, changes in functional genomics, proteomics and metabolomics already integrate the complex interplay of external exposures and internal responses. Fourth, internal dose measurements of environmental exposures might have lost the distinct signature of the relevant sources. Replacing environmental exposure assessment by exposome measurements would thereby run the risk of precluding direct links to environmental exposure measures and their sources. Without clear indications of the sources and indicators for the quantification of environmental exposure, timely implementation of mitigation strategies and their sustained surveillance is unlikely to occur.

\section{INTEGRATING THE EXPOSOME WITH TRADITIONAL AND NOVEL TOOLS FOR EXPOSURE ASSESSMENT}

We very well recognise that traditional approaches to characterising environmental exposures also have limitations. In addition, the novel approaches and the idea to introduce the concept of the exposome offer important impetus for advancements in science. We therefore propose to integrate state-of-theart exposure assessment with state-of-the-art high-throughput approaches both for characterising internal doses of xenobiotics as well as early physiological responses representing altered functional genomics, important deregulation in metabolic pathways, and changes in immune function, inflammation and endocrine regulation. Furthermore, the novel techniques are likely to open new fields of investigation, such as interaction between environment and microbiome in the gut. ${ }^{4}$ Potentially, there will be the possibility to establish libraries of untargeted metabolomics that allow future interrogation in ways currently being proposed for next-generation sequencing.

A very valuable concept embedded into the exposome paradigm is to our mind the notion to study vulnerable periods and assess environmental exposures in a life-course fashion. We strongly agree that this is a missing feature in environmental epidemiology and combined analyses of studies from different life phases need conceptual and statistical methodological developments. An integrative exposures assessment is likely to be essential for these developments and ideally should combine traditional and novel approaches.

\section{CONCLUSION}

We would like to emphasise the obligation of environmental epidemiology to provide robust evidence to assist timely and sufficient protection of vulnerable subgroups of populations from environmental hazards. Therefore, in applying the exposome concept to environmental health problems, a strong link with the external environment needs to be maintained. We recognise the emerging wealth of methods and data, and support the necessity to integrate environmental questions into research on disease mechanisms that is overturning paradigms based on technical innovations. 


\section{Competing interests None.}

Contributors The debate paper has been discussed and commented on by all authors. All authors read and approved the final version.

Provenance and peer review Commissioned; internally peer reviewed.

\section{REFERENCES}

1. Heinrich J. Influence of indoor factors in dwellings on the development of childhood asthma. Int J Hyg Environ Health 2011;214:1-25.

2. Brook RD, Rajagopalan S, Pope CA III, et al. Particulate matter air pollution and cardiovascular disease: an update to the scientific statement from the American Heart Association. Circulation 2010:121:2331-78.

3. Pope CA III, Ezzati M, Dockery DW. Fine-particulate air pollution and life expectancy in the United States. N Engl J Med 2009;360:376-86.

4. Rappaport SM. Implications of the exposome for exposure science. J Expo Sci Environ Epidemiol 2011;21:5-9.
5. Penning TM, Adamski J. Integration of steroid research: perspectives on environment factors, homeostasis in health, and disease treatment. J Steroid Biochem Mol Biol 2011;126(Suppl 1):e1-4.

6. Rakyan VK, Down TA, Balding DJ, et al. Epigenome-wide association studies for common human diseases. Nat Rev Genet 2011;12:529-41.

7. Hawkins RD, Hon GC, Ren B. Next-generation genomics: an integrative approach Nat Rev Genet 2010:11:476-86.

8. Illig T, Gieger $\mathrm{C}$, Zhai $\mathrm{G}$, et al. A genome-wide perspective of genetic variation in human metabolism. Nat Genet 2010:42:137-41.

9. Vineis $\mathbf{P}$, Khan AE, Vlaanderen J, et al. The impact of new research technologies on our understanding of environmental causes of disease: the concept of clinical vulnerability. Environmental Health 2009:8:54.

10. Rappaport SM, Smith MT. Epidemiology. Environment and disease risks. Science 2010:330:460-1.

11. Wild CP. Complementing the genome with an "exposome": the outstanding challenge of environmental exposure measurement in molecular epidemiology. Cancer Epidemiol Biomarkers Prev 2005:14:1847-50.

12. European Perspectives on Environmental Burden of Disease. Estimates for Nine Stressors in Six European Countries. Helsinki: THL, 2011.

\section{DIFFERENTIAL DIAGNOSIS}

\section{Trustworthy guidance on your iPhone}
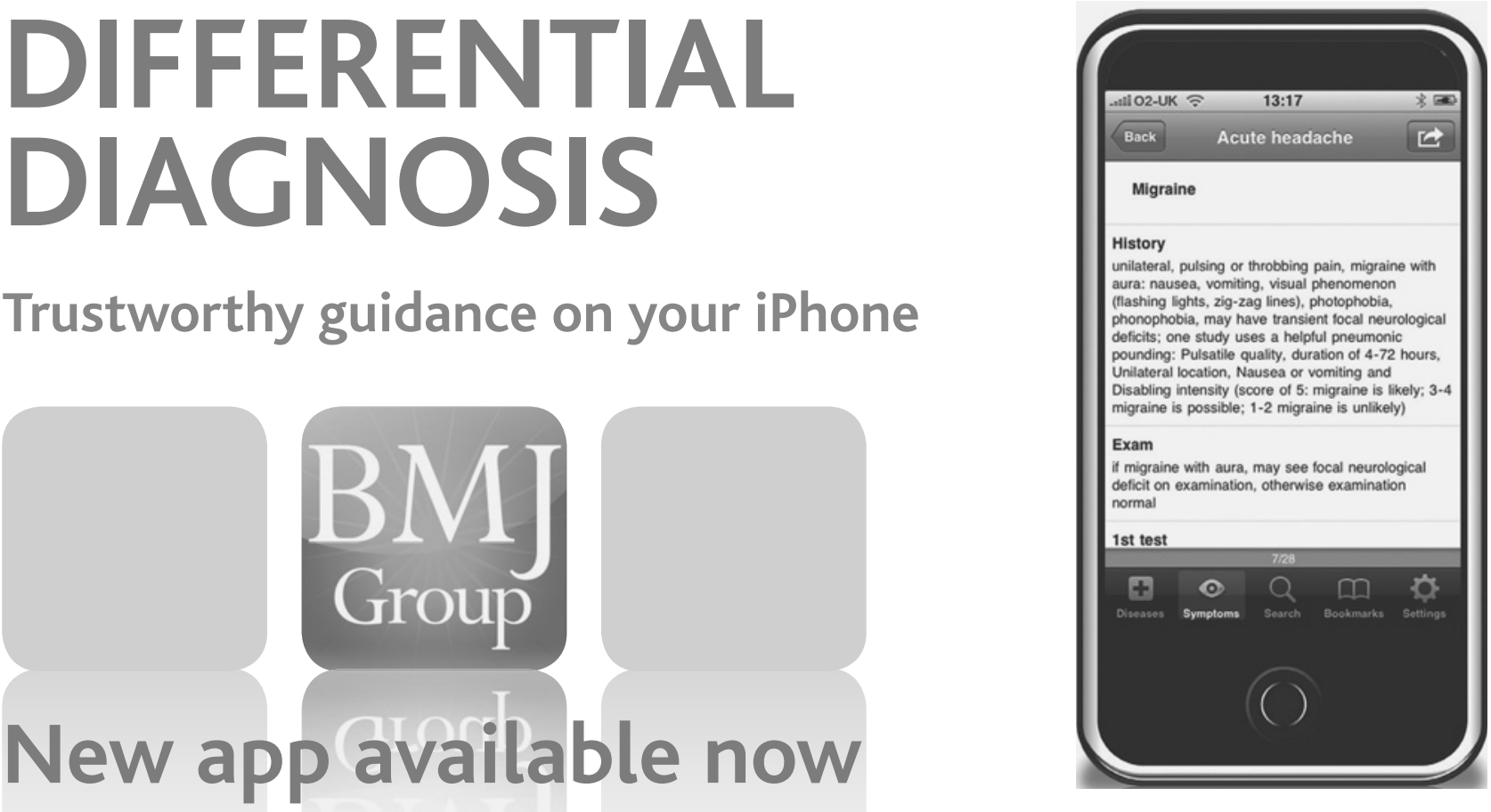

\section{Find out more at bestpractice.bmj.com/differentials}

